\title{
Sensitive Micellar Electrokinetic Chromatography-Laser-Induced Fluorescence Method To Analyze Chiral Amino Acids in Orange Juices
}

\author{
Carolina Simó, ${ }^{\dagger}$ Coral Barbas, ${ }^{\ddagger}$ And Alejandro Cifuentes $*, \dagger$ \\ Institute of Industrial Fermentations (CSIC), Juan de la Cierva 3, 28006 Madrid, Spain, and \\ Departamento de Ciencias Básicas, Facultad de CC Experimentales y Técnicas, Universidad San \\ Pablo-CEU, Urbanización Montepríncipe, 28668 Boadilla del Monte, Madrid, Spain
}

\begin{abstract}
In this work a new method to detect the existence of chiral amino acids in orange juice is presented. The method employs $\beta$-cyclodextrins and micellar electrokinetic chromatography with laser-induced fluorescence (MEKC-LIF) to separate and detect L- and D-amino acids (L-aa and D-aa) previously derivatized with fluorescein isothiocianate (FITC). A systematic optimization of the chiral-MEKC conditions is done bringing about in less than $20 \mathrm{~min}$ a good separation of the main amino acids found in orange juice (i.e., Pro, Asp, Ser, Asn, Glu, Ala, Arg, and the nonchiral GABA, i.e., $\gamma$-aminobutyric acid). Using this procedure, the analysis time reproducibility for the 15 standard compounds (L-aa, D-aa, and GABA) has been determined to be better than $0.2 \%(n=5)$ for the same day and better than $0.7 \%(n=15)$ for three different days. Corrected peak area reproducibility is somewhat lower, providing values better than $3.3 \%(n=5)$ for the same day and $6.9 \%(n=15)$ for three different days. The limit of detection using this procedure was determined to be 0.86 attomoles for L-Arg. The optimized FITC derivatization method allows the easy and straightforward detection of amino acids in orange concentrates and juices (i.e., only centrifugation of diluted samples for 5 min is needed prior to their derivatization). D-Ala, D-Asp, D-Arg, and D-Glu were determined in orange juices and orange concentrates from different geographical origins using this new method. Moreover, the effect of different temperature treatments $\left(50,92\right.$, and $\left.150^{\circ} \mathrm{C}\right)$ on the content of $\mathrm{D}$-aa in orange juice was evaluated.
\end{abstract}

KEYWORDS: Enantiomers; FITC; derivatization; LIF; juice; fruits; micellar electrokinetic chromatography; MEKC; amino acids

\section{INTRODUCTION}

The fruit juice industry has become one of the most important agricultural businesses in the world. Fruit juice trade exceeds $\$ 10$ billion per year and is dominated by citrus juice (1). Because of this economic impact, the adulteration of orange juice is an important issue that demands development of new analytical procedures that are able to detect the increasingly sophisticated adulteration procedures tailored to defeat detection methods.

A powerful detection method is the enantiomer-selective analysis of amino acids, which can provide important information on both adulteration and quality of orange juices. Thus, it has been shown that high-quality orange juice contains L-amino acids exclusively (2), whereas juices of inferior quality also contain some D-amino acids (1). Moreover, this chiral procedure can detect adulteration done with addition of synthetic amino acids or even mixtures of fruits (3). Up to now, GC and HPLC

* Corresponding author. Phone: 34-91-5622900 (Ext 387). Fax: 34 91-5644853. E-mail: acifuentes@ifi.csic.es.

Institute of Industrial Fermentations (CSIC)

$\doteqdot$ Universidad San Pablo-CEU. techniques have been used to carry out this type of enantiomeric separation $(2,3)$, providing in some cases unequivocal results. However, these techniques generally use expensive chiral columns, the procedures for sample preparation are frequently laborious and time-consuming (2), and separations may be lengthy (4). Also, in some GC procedures the derivatizing procedure does not work for some basic amino acids such as arginine (5). Considering these drawbacks of the present techniques for the chiral separation of amino acids and taking into account the economic impact of orange juice, new analytical procedures able to overcome these limitations would be very useful.

The remarkable possibilities of capillary electrophoresis (CE) for the separation of chiral compounds were first demonstrated by Gasmman et al. in 1985 (6), and this technique has been applied to multiple chiral separations (7). However, one of the main limitations of $\mathrm{CE}$ is the low sensitivity provided by the common detectors based on UV absorbance, mainly due to the narrow optical path-length used as detection cell (i.e., the capillary internal diameter). To overcome this limitation, the 
use of laser-induced fluorescence (LIF) together with derivatizing reagents (e.g., $o$-phthalaldehyde, OPA, or fluorescein isothicyanate, FITC) has been proposed as an alternative (812).

FITC has been frequently chosen as a fluorescent label for amino acids because its excitation wavelength matches the 488 $\mathrm{nm}$ light of the argon laser, and because the derivatives are easy to form, have good electrophoretic properties, and generate strong fluorescence signals (8). Despite these advantages, few papers have been devoted to the chiral separation of FITC-amino acids by CE. Thus, Nouadje et al. (13) have demonstrated the good possibilities of cyclodextrin-modified MEKC-LIF for the study of the racemization of FITC-L-serine. However, in that work only serine was analyzed. Perez-Ruiz et al. (14) also used FITC to derivatize and detect glutamate in beverages and foodstuffs. However, only that amino acid was detected and no chiral separation was involved. Jin et al. (15) carried out the enantiomeric separation of FITC-aa by MEKC using $\beta$ - and $\gamma$-cyclodextrin. These authors demonstrated that practically each individual amino acid could be enantiomericaly resolved when injected separately. Also, a group of seven amino acids could be well separated. However, as will be demonstrated below, these conditions did not accomplish the chiral separation of the group of 15 amino acids under study in this work. Moreover, to our knowledge there has not been a MEKC-LIF method developed to separate and to detect the derivatized $\mathrm{L}$ and $\mathrm{D}$ amino acids usually found in orange juice.

The goal of this work was to develop a MEKC-LIF method for the easy and fast determination of chiral amino acids in orange juices.

\section{MATERIALS AND METHODS}

Chemicals. All chemicals were of analytical reagent grade and used as received. Dextrin 10, $\beta$-cyclodextrin ( $\beta$-CD), $\gamma$-cyclodextrin $(\gamma$-CD) from Fluka (Buchs, Switzerland), and hydroxypropyl- $\beta$-cyclodextrin and dimethyl- $\beta$-cyclodextrin (both from Beckman Instruments, Fullerton, CA) were used as chiral selectors. These selectors, together with sodium dodecyl sulfate (SDS) from Acros Organics (Morris Plains, NJ) and boric acid from Riedel-De Haën (Seelze, Germany) were used for the CE running buffers at the different concentrations and $\mathrm{pHs}$ indicated. A water solution containing $1 \mathrm{M}$ sodium hydroxide from Panreac Quimica S. A. (Barcelona, Spain) was used to adjust the $\mathrm{pH}$ of the buffers. The buffers were stored at $4{ }^{\circ} \mathrm{C}$ and warmed at room temperature before use. Distilled water was deionized by using a Milli-Q system (Millipore, Bedford, MA).

Fluorescein isothiocyanate (FITC, from Fluka, Buchs, Switzerland) dissolved in acetone HPLC grade (Scharlau, Spain) was used to derivatize the amino acids at the different concentrations indicated.

L-Amino and D-amino acids and $\gamma$-aminobutyric acid (all from Sigma, St. Louis, MO) were directly dissolved in Milli-Q water at the concentrations indicated and derivatized as indicated below.

Samples and Thermal Treatments. Eight different samples (juices and concentrates) from orange were studied in this work. Namely, two orange concentrates ( $\mathrm{C} 1$ from Brazil and $\mathrm{C} 2$ from Florida) and four pasteurized juices (P1, P2, P3, and P4 from Spain) were studied. All of the orange samples were a gift from Nufri, Badajoz, Spain. One commercial juice (CJ) from orange concentrate was bought at a local market and one fresh juice (FJ) was obtained from Navelina oranges (Citrus sinensis, L. Osbeck). The fresh orange juice was freshly squeezed and used after filtration $(0.5-\mathrm{mm}$ filter) to remove pulp. This juice was treated under different temperatures and times as indicated below. Orange concentrates $\mathrm{C} 1$ and $\mathrm{C} 2$ (both $65^{\circ} \mathrm{Brix}$ ) were diluted with water till obtaining a Brix degree of ca. 10. The Brix degrees were measured with a hand refractometer from Atago (Japan). The four pasteurized juices (P1-P4), the commercial juice (CJ) from concentrate, and the fresh juices (FJ) have a Brix degree of about 10 and were directly used. All samples were centrifuged for 5 min at $10000 \mathrm{rpm}$. and the supernatant was used for derivatization.

Three thermal treatments of the fresh orange juice were studied. The first one, a pasteurization procedure, used a water bath (Tectron 3473100 , P-Selecta S. A., Spain) at $92{ }^{\circ} \mathrm{C}$ for $1 \mathrm{~min}$. The second treatment (concentration under soft conditions) used a rotary evaporator (Rüchi, Switzerland) at $50{ }^{\circ} \mathrm{C}$ for 60 min plus vacuum until $46.2^{\circ} \mathrm{Brix}$ was obtained. The third treatment (concentration under hard conditions) used a hotplate (RCT basic, IKA Labortechnik, Germany) at $150{ }^{\circ} \mathrm{C}$ for $80 \mathrm{~min}$ at atmospheric pressure until $46.8^{\circ} \mathrm{Brix}$ was obtained. Prior to their centrifugation, the two concentrated samples were diluted to obtain a similar ${ }^{\circ}$ Brix as the fresh and pasteurized juices (ca. 12).

Derivatization Procedure. The test sample containing L- and D-amino acids plus $\gamma$-aminobutyric acid was prepared by dissolving each amino acid in water to obtain a final concentration similar to that expected in the real samples (i.e., $1.5-5.7 \mathrm{mM})(1)$.

Different conditions for FITC derivatization of amino acids from orange samples were tested and they are discussed in the text. The optimum derivatization procedure consisted of mixing an aliquot of $625 \mu \mathrm{L}$ of the supernatant from orange juice (or the amino acid standard solution) with $1375 \mu \mathrm{L}$ of water and $7 \mathrm{~mL}$ of $355 \mathrm{mM}$ borate buffer. This mixture was adjusted to $\mathrm{pH} 10$ by adding $1 \mathrm{M}$ sodium hydroxide. Water was added to a final volume equal to $10 \mathrm{~mL}$. This final dissolution $(200 \mu \mathrm{L})$ was mixed with $50 \mu \mathrm{L}$ of a $30 \mathrm{mM}$ FITC solution in acetone. The reaction took place overnight in darkness at room temperature. After derivatization, test samples, and samples from orange concentrates and juices, were diluted with water $(1: 200, \mathrm{v} / \mathrm{v})$ prior to their injection in the CE-LIF instrument.

CE Conditions. Preliminary analyses were carried out in a P/ACE 2050 CE apparatus (Beckman Instruments, Fullerton, CA) equipped with an UV-Vis detector at $200 \mathrm{~nm}$. A PACE-MDQ equipped with an $\mathrm{Ar}+$ laser at $488 \mathrm{~nm}$ (excitation wavelength) and $520 \mathrm{~nm}$ (emission wavelength) from Beckman was used to detect FITC-amino acids. Bare fused-silica capillaries with 50- $\mu \mathrm{m}$ i.d. were purchased from Composite Metal Services (Worcester, England) and used with the lengths indicated in each case. Injections were made at the anodic end using $\mathrm{N}_{2}$ pressure of $0.5 \mathrm{psi}$ for a given time $(1 \mathrm{psi}=6894.76 \mathrm{~Pa})$. The P/ACE $2050 \mathrm{CE}$ instrument was controlled by a PC running the System GOLD software, and the PACE-MDQ was controlled by a PC running the 32 Karat software; both software packages were from Beckman.

Before first use, any new capillary was preconditioned by rinsing with $0.1 \mathrm{M} \mathrm{NaOH}$ for $30 \mathrm{~min}$. Between injections, capillaries were rinsed with water for $2 \mathrm{~min}$ and with the separation buffer for $3 \mathrm{~min}$. As will be shown below, the optimun running buffer consisted of 100 $\mathrm{mM}$ sodium tetraborate, $30 \mathrm{mM}$ SDS at $\mathrm{pH}$ 9.4. At the end of the day, the capillary was rinsed with deionized water for $5 \mathrm{~min}$ and stored overnight with water inside.

\section{RESULTS AND DISCUSSION}

A group of seven chiral amino acids (Pro, Asp, Ser, Asn, Glu, Ala, and Arg) plus the nonchiral amino acid GABA was initially selected to carry out this study. This selection was based on the fact that these eight amino acids are responsible for more than $90 \%$ of the amino acidic content in orange juices $(1,14)$. The rest of the amino acids usually found in orange juices are present in percentages lower than $10 \%(1)$. Because a total of 14 enantiomers plus GABA were in our study, the profiles obtained from these 15 compounds should be sufficiently characteristic to define a juice (e.g., to determine whether D-amino acids are naturally occurring substances in orange or whether they are released under specific fruit processing procedures) allowing detection of certain types of adulteration. Some of these detectable adulterations would be, for instance, the addition of inexpensive racemic amino acids (e.g., glutamic acid) or the addition of protein hydrolysates to increase the total amino acid content (1). Also, the detection of D-amino acids in orange juice can be indicative of a possible microbial spoilage or related to juices of unsatisfactory quality (5). 


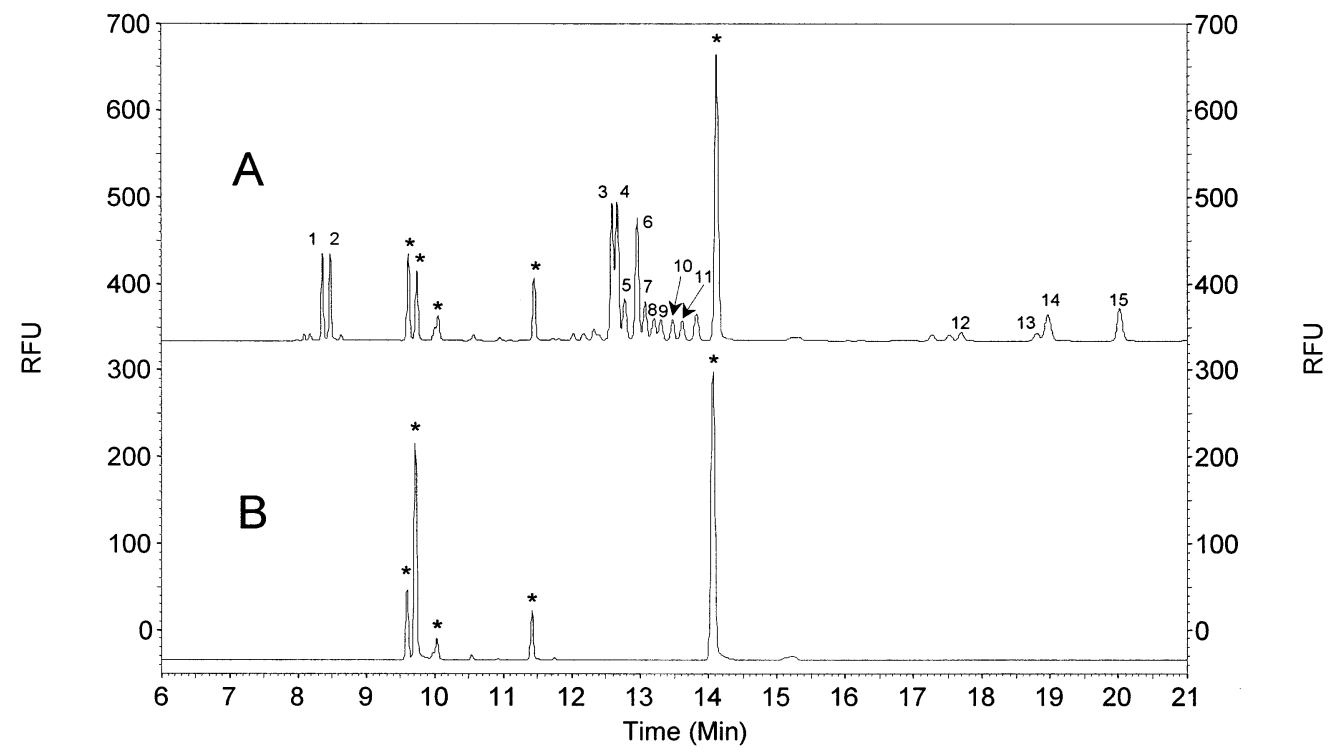

Figure 1. (A) Chiral MEKC-LIF separation of standard FITC-amino acids. (B) Electropherogram of the peaks coming from nonreacting FITC (marked with asterisks). Conditions were the following: capillary, $\mathrm{I}_{\mathrm{d}}=40 \mathrm{~cm}, \mathrm{I}_{\mathrm{t}}=50 \mathrm{~cm}$ with $50 \mu \mathrm{m}$ i.d.; running buffer, $100 \mathrm{mM}$ sodium tetraborate, $30 \mathrm{mM}$ SDS at pH 9.4 with $20 \mathrm{mM} \beta$-CD; capillary temperature, $15^{\circ} \mathrm{C}$; run voltage, $20 \mathrm{kV}$; injection at $0.5 \mathrm{psi}$ for $5 \mathrm{~s}$ of FITC derivatized (1) D-Arg, (2) L-Arg, (3) L-Pro, (4) D-Pro, (5) D-Asn, (6) GABA, (7) L-Asn, (8) D-Ser, (9) D-Ala, (10) L-Ser, (11) L-Ala, (12) D-Glu, (13) L-Glu, (14) D-Asp, (15) L-Asp, (*) peak from FITC. Concentration of amino acids injected $(\mu \mathrm{M})$ : D,L-Arg (1.06), D,L-Pro (1.9), GABA (0.65), Asn (0.77), Ser (0.39), Ala (0.27), Glu (0.17) and Asp (0.59). LIF detection at $488 \mathrm{~nm}$ (excitation wavelength) and $520 \mathrm{~nm}$ (emission wavelength).

Development of the Chiral MEKC-LIF Method. Initially, the 15 amino acidic forms derivatized with FITC were injected using the two optimized MEKC conditions given by Jin et al. in their work (15). A good separation of the four enantiomers L,D-Asp and L,D-Glu, was obtained in about $20 \mathrm{~min}$ (data not shown). However, a group of 9 compounds plus the nonreacting FITC comigrated in all the cases. Therefore, a further optimization of this method was tried.

First, different chiral selectors were tested to separate the group of FITC-amino acids under study. Namely, $\beta$-CD, hydroxypropyl- $\beta$-CD (Hy- $\beta$-CD), dimethyl- $\beta$ - $\mathrm{CD}$, and Dextrin 10 (D10) were dissolved in different concentrations into the run buffer. Neither Hy- $\beta$-CD or dimethyl- $\beta$-CD allowed the separation of all the L- and D-amino acids derivatized with FITC in a single run. Dimethyl- $\beta$-CD gave the worst results, as with this chiral selector at the two concentrations tested (10 and 30 $\mathrm{mM}$ ) no enantiomeric separation for the group of amino acids under study could be achieved. The chiral selector Hy- $\beta$-CD was tested at the concentrations of 10 and $20 \mathrm{mM}$. Under the best conditions (i.e., $10 \mathrm{mM}$ ), Hy- $\beta$-CD did not provide the chiral separation for Arg and Pro; moreover, at these conditions four enantiomers corresponding to D,L-Ser and D,L-Ala comigrated in three peaks. This buffer containing $10 \mathrm{mM} \mathrm{Hy-} \beta$-CD was mixed with 5 and $20 \mathrm{mM} \beta$-CD. Although an improvement in the chiral resolution of $\mathrm{D}$ - and L-Arg was obtained by adding $5 \mathrm{mM} \beta$-CD, the other mentioned difficulties remain. A linear maltodextrin, Dextrin 10, was then used for the separation of FITC-aa (to our knowledge for the first time). The use of this dextrin provided a baseline separation of the derivatized L- and D-Arg, Ala, Glu, Asn, and Ser. However, the enantiomers of Pro and Asp could not be separated. The resolution of enantiomers of Pro was improved by increasing the percentage of Dextrin 10, while no improvement was detected for the separation between D-Asp and L-Asp. Besides, the injection of the 15 amino acidic forms all together gave as result the comigration of several compounds; namely, the enantiomers of Pro, Asn, Ser, and Ala were observed to comigrate. Further attempts to improve this separation (e.g., modifying $\mathrm{pH}$, increasing concentration of Dextrin 10, adding other different dextrins to the buffer containing Dextrin 10) did not provide satisfactory results.

A systematic study was then done using $\beta$-CD as chiral selector. To do this, the effect brought about by the use of different $\beta$-CD concentrations (from 10 to $25 \mathrm{mM}$ in $5-\mathrm{mM}$ steps), SDS concentrations $(15,30,60$, and $100 \mathrm{mM})$, buffer $\mathrm{pH}$ (from 9.2 to 10.8 in 0.2 -unit steps), and temperature of separation (from $15^{\circ} \mathrm{C}$, the minimum achieved by the apparatus, to $30{ }^{\circ} \mathrm{C}$ in $5-{ }^{\circ} \mathrm{C}$ steps) were analyzed. Also, $\beta$-CD was mixed with several chiral selectors ( $\gamma-\mathrm{CD}, \mathrm{Hy}-\beta-\mathrm{CD}$, and Dextrin 10 ) and acetonitrile ( 2 and $5 \%$ ) as organic modifier.

From this study, the best separation conditions were found to be the following: $100 \mathrm{mM}$ sodium tetraborate, $20 \mathrm{mM} \beta-\mathrm{CD}$, $30 \mathrm{mM}$ SDS at $\mathrm{pH} 9.4$, and a temperature of $15^{\circ} \mathrm{C}$. As can be seen in Figure 1A, an acceptable separation of the 15 amino acids is achieved in less than $20 \mathrm{~min}$ using these optimized conditions. Under these conditions, the worst separation was obtained for D- and L-Pro (peaks 3 and 4) with a resolution value equal to 0.6 (calculated as $\left(t_{\mathrm{D}-\text { Pro }}-t_{\mathrm{L}-\text { Pro }}\right) / w_{\mathrm{D}-\text { Pro }}$, where $t_{\mathrm{D}-\text { Pro }}$ and $t_{\mathrm{L}-\text {-Pro }}$ are the migration times for D-Pro and $\mathrm{L}-$ Pro, and $\mathrm{w}_{\mathrm{D}}-$ Pro is the baseline peak width for the last migrating peak, i.e., D-Pro in this case). Moreover, it can be seen by comparing Figure 1A with Figure 1B that peaks coming from nonreacting FITC or impurities (marked with asterisks) do not interfere with the separated amino acids.

Reproducibility Study. The reproducibility of this MEKCLIF method was checked in terms of analysis time and peak area using the conditions of Figure 1. Results for three representative amino acids (Arg, Ser, and Asp, corresponding to the first, middle, and last migrating $\mathrm{L}$ enantiomers, respectively) are given in Table $\mathbf{1}$. The analysis time reproducibility was better than $0.2 \%$ (given as relative standard deviation, $\%$ RSD, for $n=5$ injections) for the same day and better than $0.7 \%$ for three different days ( $n=15$ injections). As expected, reproducibility for corrected peak areas (area/time) was lower 
Table 1. Reproducibility of Migration Times $(t)$ and Corrected Peak Areas $\left({ }^{A} / t\right)$ Obtained for FITC-L-Arg, FITC-L-Ser, and FITC-L-Asp for the Same day (5 Injections) and Three Different days (15 Injections) $)^{a}$

\begin{tabular}{|c|c|c|c|c|c|c|c|c|}
\hline & \multicolumn{4}{|c|}{ same day $(n=5)$} & \multicolumn{4}{|c|}{ three days $(n=15)$} \\
\hline & $\overline{t(\min )}$ & $\% \mathrm{RSD}_{t}$ & $A / t^{b}(\mathrm{RFU} / \mathrm{min})$ & $\% \mathrm{RSD}_{A / t}$ & $t(\min )$ & $\% \mathrm{RSD}_{t}$ & $A / t$ (RFU/min) & $\% \mathrm{RSD}_{A / t}$ \\
\hline L-Arg & 8.4 & 0.1 & 23337 & 1.9 & 8.4 & 0.2 & 23756 & 4.7 \\
\hline L-Ser & 13.5 & 0.2 & 6281 & 2.2 & 13.5 & 0.3 & 6404 & 5.9 \\
\hline L-Asp & 19.9 & 0.1 & 2878 & 3.3 & 20.0 & 0.7 & 2990 & 6.9 \\
\hline
\end{tabular}

${ }^{a}$ All the conditions are as in Figure 1. ${ }^{b A} / t$ is the corrected peak area where $A$ refers to the peak area and $t$ is the migration time of each amino acid.

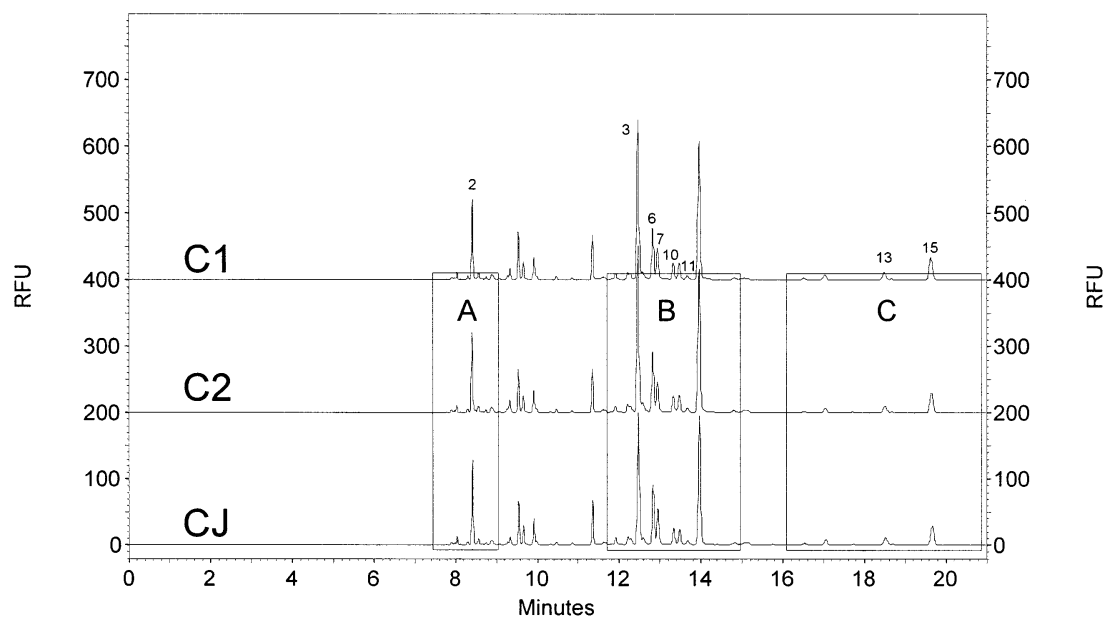

A

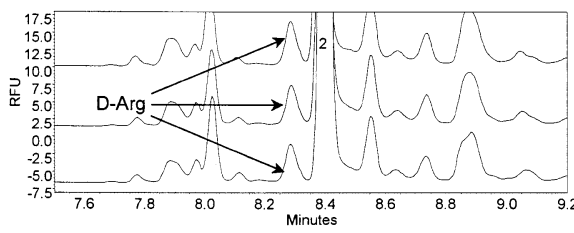

B

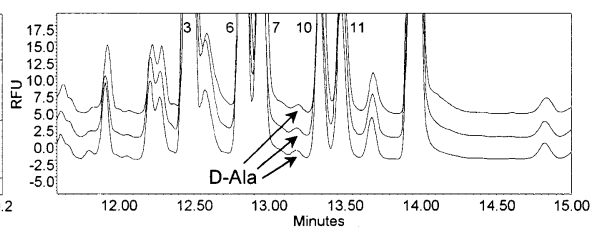

C

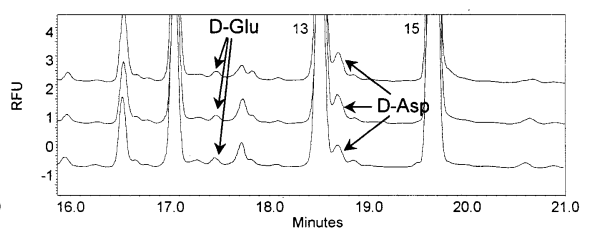

Figure 2. FITC-amino acids in orange juices. (A) Orange concentrate $\mathrm{C1}$, $(\mathrm{B})$ orange concentrate $\mathrm{C} 2$, (C) orange concentrate $\mathrm{CJ}$. Separation conditions as in Figure 1.

than that for analysis times, providing values better than $3.3 \%$ $(n=5)$ for the same day and $6.9 \%(n=15)$ for three different days.

FITC-Amino Acids Derivatization and Application to Orange Juices. As mentioned above, the existing GC and HPLC methods for detecting chiral amino acids in orange fruits are usually based on laborious and time-consuming sample preparation protocols (2). Therefore, the development of a direct procedure to obtain FITC-aa from the centrifuged orange samples was attempted. It was observed that the $\mathrm{pH}$ of the supernatants from different orange samples could vary from 3.48 (pasteurized juice) to 3.86 (concentrate), which was observed to modify the yield of reaction with FITC during our study. Different parameters were then tested in order to obtain a sensitive and robust derivatizing procedure independent of the type of sample. To do this, different concentrations of FITC (from 1 to $30 \mathrm{mM}$ ), different sample dilutions (from 5 to 30), and different $\mathrm{pHs}$ (from 7 to 11) and ionic strengths (from 0 to $400 \mathrm{mM}$ ) of the sodium tetraborate buffer used in the reaction, were tested. It could be seen that the higher the FITC concentration, the higher the signal obtained $(30 \mathrm{mM}$ is already above the solubility limit of this compound in acetone, therefore, no higher concentration was tested). A sample dilution between 10 and 30 brings about a proportional decrease of the signal for all amino acids. However, at dilutions lower than 10 the derivatization reaction is mainly favored for Arg and Pro. Concentration of the tetraborate buffer added to the orange sample and $\mathrm{pH}$ of the mixture prior to its derivatization was also observed to modify the derivatization yield. Therefore, the optimum conditions for maximum signal consisted of (for details see Material and Methods section) the following: mixing an aliquot of the supernatant from orange juice with water, using borate buffer, adjusting till $\mathrm{pH} 10$ with sodium hydroxide, and adding FITC to a diluted aliquot of that final dissolution.

Under these conditions, separations such as the ones shown in Figure 2 were obtained for the different orange juice samples. As can be seen, L-Arg (peak 2), L-Pro (peak 3), GABA (peak 6), L-Asn (peak 7), L-Ser (peak 10), L-Ala (peak 11), L-Glu (peak 13), and L-Asp (peak 15) can be easily detected. Moreover, L-Arg, L-Pro, and L-Asn appear to be the dominant forms, in good agreement with that observed by other authors $(1,16)$. The limit of detection of this procedure, calculated as moles of injected L-arginine needed for obtaining a signal equal to three times the signal noise, was determined to be equal to 0.86 attomoles, which seems to corroborate the usefulness of this protocol.

Interestingly, D-Arg, D-Ala, D-Glu, and D-Asp could be detected in the orange samples, as can be seen in the insets of Figure 2. The nature of these peaks was further corroborated by spiking each orange juice with known quantities of the corresponding D-amino acid, carrying out the complete derivatization protocol, and injecting them in MEKC-LIF. An example of this confirmation procedure is given in Figure 3, for which known concentrations of D-Asp were added. Thus, 
Table 2. Amino Acids Peak Areas Found in Four Pasteurized Juices (Samples P1-P4), Two Orange Concentrates (Samples C1 and C2), and One Commercial Juice from Orange Concentrate (CJ)

\begin{tabular}{|c|c|c|c|c|c|c|c|c|c|c|c|c|c|}
\hline sample & ${ }^{\circ}$ Brix & D-Arg & L-Arg & L-Pro & GABA & L-Asn & D-Ala & L-Ser & L-Ala & D-Glu & L-Glu & D-Asp & L-Asp \\
\hline P1 & 7.5 & 1326 & 20539 & 45704 & 14131 & 24447 & 347 & 7164 & 3177 & 164 & 3240 & 221 & 10090 \\
\hline P2 & 9.0 & 1364 & 21958 & 48958 & 8204 & 21398 & 349 & 6735 & 3468 & 177 & 5014 & 294 & 12861 \\
\hline P3 & 9.5 & 1601 & 27922 & 101383 & 23215 & 25765 & 317 & 9623 & 5994 & 196 & 4874 & 316 & 16117 \\
\hline P4 & 10.5 & 1617 & 43449 & 129261 & 36687 & 32621 & 847 & 12729 & 11378 & 196 & 5700 & 414 & 14155 \\
\hline C1 & 10.5 & 1498 & 27585 & 84812 & 28058 & 17958 & 658 & 10048 & 10285 & 231 & 6844 & 532 & 18967 \\
\hline C2 & 10.5 & 1252 & 24516 & 78298 & 29279 & 15327 & 623 & 8515 & 9606 & 148 & 5282 & 518 & 14209 \\
\hline CJ & 11.0 & 1470 & 31098 & 76122 & 36379 & 22261 & 585 & 10498 & 10134 & 207 & 7377 & 516 & 18243 \\
\hline
\end{tabular}

Table 3. Amino Acids Peak Areas Found in a Fresh Orange Juice (FJ), and the Same Juice after Three Different Thermal Treatments (Conditions in Text)

\begin{tabular}{|c|c|c|c|c|c|c|c|c|c|c|c|c|c|}
\hline sample & ${ }^{\circ}$ Brix & D-Arg & L-Arg & L-Pro & GABA & L-Asn & D-Ala & L-Ser & L-Ala & D-Glu & L-Glu & D-Asp & L-Asp \\
\hline FJ & 12.0 & 1681 & 30969 & 82773 & 22270 & 33477 & 435 & 11.545 & 6073 & 162 & 4334 & 367 & 16619 \\
\hline FJ pasteurized ${ }^{a}$ & 11.9 & 1774 & 34577 & 91709 & 24877 & 36764 & 736 & 10.175 & 6920 & 180 & 4699 & 404 & 18350 \\
\hline FJ concentrated & 12.0 & 1676 & 33970 & 90246 & 24196 & 36564 & 81 & 12.413 & 6925 & 183 & 4688 & 45 & 18210 \\
\hline FJ concentrated hard ${ }^{c}$ & 12.0 & 1204 & 34331 & 91803 & 25254 & 36747 & 900 & 12.918 & 6960 & 207 & 4950 & 532 & 19235 \\
\hline
\end{tabular}

${ }^{a}$ Treated for $1 \mathrm{~min}$ at $92{ }^{\circ} \mathrm{C}$ using a water bath. ${ }^{b}$ Treated for $60 \mathrm{~min}$ at $50{ }^{\circ} \mathrm{C}$ using a rotary evaporator plus vacuum until $46.2{ }^{\circ}$ Brix was obtained. ${ }^{c}$ Treated for 80 min at $150^{\circ} \mathrm{C}$ using a hotplate until $46.8^{\circ}$ Brix was obtained.

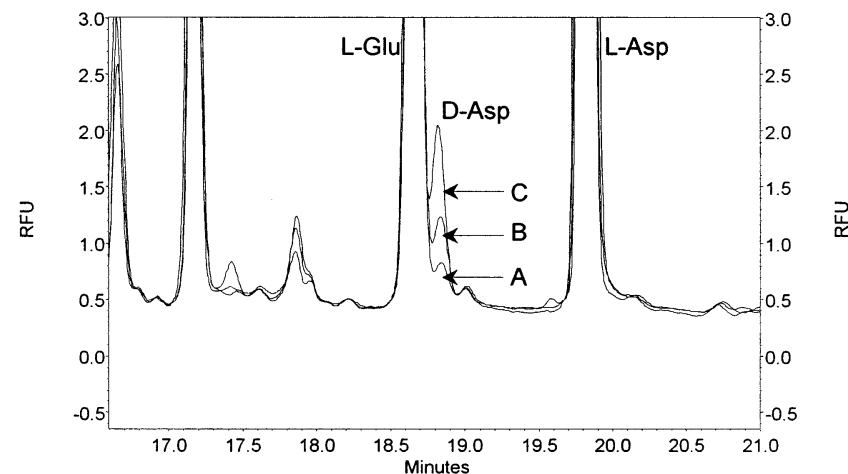

Figure 3. Electropherograms of the $\mathrm{C} 1$ orange juice $(\mathrm{A})$; $\mathrm{C} 1$ plus $1.5 \times$ $10^{-6} \mathrm{M}$ D-Asp (B); and C1 plus $4.4 \times 10^{-6} \mathrm{M}$ D-Asp (C). All the conditions as in Figure 1.

by using this procedure, the original concentration of D-Asp found in this sample was determined to be $1.7 \times 10^{-6} \mathrm{M}$. These results are in good agreement with the findings of Bruckner and Westhauser (4) and Friedman (17) regarding the existence of these four $\mathrm{D}$-aa in different fruits including oranges. Moreover, according to the literature, the quantity of D-Asp determined in orange is in the micromolar range (i.e., $8 \times 10^{-6}$ M) (4), in good agreement with the quantity determined by our procedure.

Two orange concentrates (samples $\mathrm{C} 1$ from Brazil and $\mathrm{C} 2$ from Florida), four pasteurized juices (samples P1, P2, P3, and P4 from Spain) and one commercial juice from orange concentrate $(\mathrm{CJ})$ were studied using this procedure. The peak areas obtained for the different amino acids found in these eight samples are given in Table 2. A first conclusion that can be reached from these results is the existence of the four D-amino acids (D-Arg, D-Ala, D-Glu, and D-Asp) in all orange juices independent of the geographical origin (Brazil, Florida, or Spain) and treatment of the sample (i.e., pasteurized or concentrated). Also, in the case of the four pasteurized juices it can be deduced that (except for GABA and L-Asn) the higher the Brix degree the higher the amino acid content detected in these juices.

From the differences in the quantity of D-Asp detected among pasteurized and concentrated juices of Table 2 it could be thought that this compound could be used to identify the existence of a given thermal treatment. Unfortunately, the necessary information about the variety of oranges from which these samples come from or their storage was not available. Therefore, the next experiment was carried out following two different objectives: first, to corroborate that the D-amino acids detected are naturally occurring constituents of orange juice and not due to microbial spoilage (5) or storage (18); and second, to discern if the thermal treatment of orange juices brings about racemization of amino acids and if any of the D-aa can be used as a molecular marker of such treatments. Pasteurization has been mentioned to bring about the formation of D-amino acids in fruit juices (17), although other authors have not detected a significant increase of D-Ala after heat treatment of fruit juices (18).

A fresh juice (FJ), obtained as described under Materials and Methods, was prepared and submitted to three different thermal treatments. The first one consisted of a pasteurization procedure (i.e., $92{ }^{\circ} \mathrm{C}$ for $1 \mathrm{~min}$ ) and the other two were intended to simulate soft and a hard concentration procedures (described in Materials and Methods). Results of the amino acids peak areas detected in these four samples are given in Table 3. As can be seen, D-amino acids are detected in the fresh orange juice, which seems to corroborate the natural origin of these molecules, together with the usefulness of our procedure to detect them. Moreover, from our results it seems that D-Asp and, perhaps more clearly, D-Ala, could be used as a possible molecular marker of thermal treatment of orange juices (17-21) because, as demonstrated in Table $\mathbf{3}$, the quantity of this amino acid seems to correlate with the intensity of the thermal treatment.

\section{CONCLUSIONS}

The possibilities of a new method to detect the existence of chiral amino acids in orange juice have been demonstrated. The optimized FITC derivatization conditions allows the easy and straightforward derivatization of amino acids from orange concentrates and juices (i.e., only centrifugation of diluted samples for $5 \mathrm{~min}$ is needed prior to their derivatization). The new MEKC-LIF method developed brings about the separation and detection of the main amino acids found in orange juice (i.e., Pro, Asp, Ser, Asn, Glu, Ala, Arg, and the nonchiral GABA, i.e., $\gamma$-aminobutyric acid) in less than 20 min, with good analysis time and peak area reproducibilities. D-Ala, D-Asp, D-Arg, and D-Glu were determined in orange juices and orange 
concentrates from different geographical origins using this new method. Moreover, the effect of different temperature treatments on the content of D-amino acids in orange juice was evaluated.

\section{LITERATURE CITED}

(1) Robards, K.; Antolovich, M. Methods for assessing the authenticity of orange juice. A review. Analyst 1995, 120, 1-28.

(2) Gandolfi, I.; Palla, G.; Dossena, A.; Puelli, S.; Salvadori, C. D-alanine in fruit juices: a molecular marker of bacterial activity, heat treatments and shelf life. J. Food Sci. 1994, 59, 152-154.

(3) Ooghe, W.; Kasteleyn, H.; Temmerman, I.; Sandra, P. Amino acid enantiomer separation for the detection of adulteration in fruit juices. J. High Resolut. Chromatogr. 1984, 7, 284-285.

(4) Bruckner, H.; Westhauser, T. Chromatographic determination of D-amino acids as native constituents of vegetables and fruits. Chromatographia 1994, 39, 419-426.

(5) Bruckner, H.; Lupke, M. Determination of amino acids enantiomers in orange juice by chiral phase capillary gas chromatography. Chromatographia 1991, 31, 123-128.

(6) Gassman, E.; Kuo, J. E.; Zare, R. N. Electrokinetic separation fo chiral compounds. Science 1985, 230, 813-814.

(7) Chankvetadze, B. Capillary Electrophoresis in Chiral Analysis; John Willey \& Sons: Chichester, England, 1997.

(8) Cheng, Y.-F.; Dovichi, N. J. Subattomole amino acid analysis by capillary zone electrophoresis and laser-induced fluorescence. Science 1988, 242, 562-564.

(9) Issaq, H. J.; Chan, K. C. Separation and detection of amino acid and their enantiomers by capillary electrophoresis: a review. Electrophoresis 1995, 16, 467-481.

(10) Wan, H.; Blomerg, L. G. Chiral separation of amino acids and peptides by capillary electrophoresis. J. Chromatogr. A 2000, 875, 43-88.

(11) Engstroem, A.; Andersson, P. E.; Jossefsson, B.; Pfeffer, D. Determination of 2-(9-Anthryl)ethyl Chloroformate-labeled amino acids by capillary electrophoresis and liquid chromatography with absorbance or fluorescence detection. Anal. Chem. 1995, 67, 3018-3022.

(12) Kaneta, T.; Shiba, H.; Imakasa, T. Determination of cyaninelabeled amino acids enantiomers by cyclodextrin-modified capillary gel electrophoresis combined with laser induced fluorescence detection. J. Chromatogr. A. 1998, 805, 295-300.
(13) Nouadge, G.; Nertz, M.; Couderc, F. Study of racemization of L-serine by cyclodextrin-modified micellar electrokinetic chromatography and laser-induced fluorescence detection. J. Chromatogr. A. 1995, 716, 331-334.

(14) Pérez-Ruiz, T.; Martínez-Lozano C.; Bravo, E. Analysis of glutamate in beverages and foodstuffs by capillary electrophoresis with laser-induced fluorescence detection Chromatographia 2000, 52, 599-602.

(15) Jin, L. J.; Rodriguez, I.; Li, S. F. Y. Enantiomeric separation of amino acids derivatized with fluoresceine isothiocyanate isomer I by micellar electrokinetic chromatography using $\beta$ - and $\gamma$-cyclodextrins as chiral selectors. Electrophoresis 1999, 20, $1538-1545$.

(16) Kimball, D. A.; Norman, S. I. Processing affects during commercial debittering of California Navel orange juice. J. Agric. Food Chem. 1990, 38, 1396-1400.

(17) Friedman, M. J. Chemistry, nutrition, and micobiology of D-amino acids. J. Agric. Food Chem. 1999, 47, 3457-3479.

(18) Gandolfi, I.; Palla, G.; Delprato, L.; De Nisco, F.; Marchelli, R.; Salvadori, C. D-amino acids in milk as realted to heat treatments and bacterial activity. J. Food Sci. 1992, 57, 377379.

(19) Del Castillo, M. D.; Santa-Maria, G.; Pueyo, E.; Corzo, N.; Olano, A. Differences in amino acid composition in commercial orange juices. J. Agric. Food Chem. 1998, 46, 2329-2331.

(20) Del Castillo, M. D.; Corzo, N.; Olano, A. Early stages of Maillard reaction in dehydrated orange juice. J. Agric. Food Chem. 1999, 47, 4388-4390.

(21) Del Castillo, M. D.; Villamiel, M.; Olano, A.; Corzo, N. Use of 2-Furoylmethyl derivatives of GABA and Arginine as indicators of the initial steps of Maillard reaction in orange juices. J. Agric. Food Chem. 2000, 48, 4217-4220.

Received for review April 2, 2002. Revised manuscript received June 18, 2002. Accepted June 18, 2002. This work was supported by a CICYT project (ALI99-1188). C.S. thanks Consejería de Educación y Cultura (Comunidad de Madrid) for a fellowship.

JF0203848 